\title{
A noninvasive algorithm to exclude pre-capillary pulmonary hypertension
}

\author{
D. Bonderman*, P. Wexberg*, A.M. Martischnig*, H. Heinzl”, M-B. Lang*, \\ R. Sadushi*, N. Skoro-Sajer* and I.M. Lang*
}

ABSTRACT: Current guidelines recommend right heart catheterisation (RHC) in symptomatic patients at risk of pre-capillary pulmonary hypertension (PH) with echocardiographic systolic pulmonary artery pressures $\geqslant 36 \mathrm{mmHg}$. Growing awareness for $\mathrm{PH}$, a high prevalence of postcapillary PH and the inability to distinguish between pre- and post-capillary PH by echocardiography have led to unnecessary RHCs. The aim of our study was to assess whether standard noninvasive diagnostic procedures are able to safely exclude pre-capillary $\mathrm{PH}$.

Data from 251 patients referred for suspicion of pre-capillary PH were used to develop a noninvasive diagnostic decision tree. A prospectively collected data set of 121 consecutive patients was utilised for temporal validation.

According to the decision tree, patients were stratified by the presence or absence of an electrocardiographic right ventricular strain pattern (RVS) and serum N-terminal brain natriuretic peptide (NT-proBNP) levels below and above $80 \mathrm{pg} \cdot \mathrm{mL}^{-1}$. In the absence of RVS and elevated NTproBNP, none of the patients in the prospective validation cohort were diagnosed with pre-capillary PH by RHC. Combining echocardiography with the diagnostic algorithm increased specificity to $19.3 \%(p=0.0009)$, while sensitivity remained at $100 \%$.

Employing ECG and NT-proBNP on top of echocardiography helps recognise one false positive case per five patients referred with dyspnoea and echocardiographic suspicion of $\mathrm{PH}$, while not missing true pre-capillary $\mathrm{PH}$.

KEYWORDS: Diagnostic procedures, pulmonary hypertension

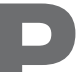

re-capillary pulmonary hypertension $(\mathrm{PH})$ is a severe condition leading to right heart failure and death within 2-3 yrs after diagnosis, if left untreated [1]. While idiopathic pulmonary arterial hypertension (PAH) is rare, associated forms of PAH [2] are more common and may be triggered by collagen vascular disease, appetite suppressants [3], HIV-infection [4], increased shear stress and hypoxia [2, 5-7]. A series of medical conditions, including infection, immune disorders, inflammatory bowel disease and permanent venous catheters [8, 9], predispose to chronic thromboembolic pulmonary hypertension (CTEPH) [10].

According to international guidelines [2], which were recently updated [11-13], invasive haemodynamic measurement by right heart catheterisation (RHC) is recommended in patients with clinical suspicion of pre-capillary $\mathrm{PH}$ if systolic pulmonary artery pressure ( $P$ pa,sys) by transthoracic echocardiography (TTE) is $\geqslant 36 \mathrm{mmHg}$.
An invasively measured pulmonary capillary wedge pressure of $15 \mathrm{mmHg}$ has been used to discriminate between pre- and post-capillary pulmonary pressure elevation which mostly occurs as a consequence of left-sided heart disease [14]. The growing awareness for $\mathrm{PH}$, a high prevalence of post-capillary $\mathrm{PH}$ [15] and the inability of TTE to distinguish between pre- and post-capillary $\mathrm{PH}$ have necessitated invasive haemodynamic measurements for exclusion. Furthermore, Doppler echocardiography is frequently inaccurate in estimating Ppa,sys [16]. Given a low specificity of $\mathrm{PH}$ symptoms and $P$ pa,sys elevations by TTE, the decision to either proceed with RHC or withhold further invasive testing is a common clinical problem, especially in high-volume $\mathrm{PH}$ referral centres [17]. Although rare [18], complications of RHC may be ventricular tachyarrhythmia, vascular or ventricular perforation, bleeding, pneumothorax and even death [19]. However, a restrictive use of $\mathrm{RHC}$ may delay a timely diagnosis and treatment [20].
AFFILIATIONS

*Dept of Cardiology, and

${ }^{\#}$ Core Unit for Medical Statistics and Informatics, Medical University of Vienna, Vienna, Austria.

CORRESPONDENCE

D. Bonderman

Dept of Internal Medicine II, Division of Cardiology

Medical University of Vienna

Währinger Gürtel 18-20

1090 Vienna

Austria

E-mail: diana.bonderman@

meduniwien.ac.at

Received:

June 102010

Accepted after revision:

July 282010

First published online:

Aug 062010 
Apart from TTE, 12-lead electrocardiography (ECG), serum $\mathrm{N}$-terminal brain natriuretic peptide (NT-proBNP) and lung function tests with blood gas analysis have been recommended in patients who present with dyspnoea. Typical ECG signs of pre-capillary $\mathrm{PH}$ are right ventricular hypertrophy and strain (RVH and RVS, respectively), and signs of right atrial dilation such as P-pulmonale. The role of ECG for the diagnosis of PH has been investigated in a large US registry initiated in the early 1980s. RicH et al. [21] reported that electrocardiographic $\mathrm{RVH}$ was present in $87 \%$ and right axis deviation in $79 \%$ of patients with idiopathic PH. Elevated serum NT-proBNP [22] and hypocapnia [23] have been established as independent markers of mortality in $\mathrm{PH}$. However, the combined diagnostic use of these noninvasive tests has not been validated. Therefore, we tested the ability of standard noninvasive diagnostic procedures to correctly identify or exclude pre-capillary $\mathrm{PH}$ in patients referred for clinical suspicion of $\mathrm{PH}$ and echocardiographic $P$ pa,sys $\geqslant 36 \mathrm{mmHg}$. We used a retrospective data set of 251 consecutive individuals and constructed a simple noninvasive diagnostic algorithm. In a prospective temporal validation study enrolling 121 new consecutive patients, the incremental diagnostic value of the combined use of TTE, ECG and NT-proBNP over TTE alone was confirmed.

\section{METHODS}

\section{Setting and study design}

The study was approved by the Ethics Committee of the Medical University of Vienna (Vienna, Austria), and written informed consent was collected from prospectively enrolled patients. The study was performed at the Pulmonary Hypertension Unit of the Medical University of Vienna as a tertiary referral centre for $\mathrm{PH}$.

\section{Retrospective study}

Between January 2002 and April 2007, 462 patients were referred for the evaluation of PH. TTE, 12-lead ECG, serum NT-proBNP and lung function tests including arterial carbon dioxide tension $\left(\mathrm{Pa}, \mathrm{CO}_{2}\right)$ were performed upon admission in each patient. Patients with echocardiographic $P$ pa,sys $<36 \mathrm{mmHg}(\mathrm{n}=80)$ were not considered for invasive haemodynamic assessment. Patients carrying pacemakers or implantable cardioverters/defibrillators $(n=12)$ were excluded because of the inapplicability of standard ECG criteria. 71 patients were excluded because of severe valvular disease, congenital malformations and/or severely impaired left ventricular function. Of the 299 patients who were considered appropriate study candidates, 48 were excluded because of incomplete data, leaving 251 patients for analysis. Based on the invasive haemodynamic evaluation by RHC, the diagnosis "pre-capillary $\mathrm{PH}^{\prime}$ or "no pre-capillary $\mathrm{PH}^{\prime}$ was made.

\section{Prospective study}

For temporal validation of the diagnostic algorithm constructed from the retrospective data set, we enrolled consecutive patients referred between June 2007 and October 2008. From a total of 222 individuals, 101 were excluded because of valvular heart disease $(n=29)$, left ventricular dysfunction $(n=8)$, congenital heart disease $(n=8)$, pacemaker $(n=17)$ and an echocardiographic $P$ pa,sys $<36 \mathrm{mmHg}(\mathrm{n}=30)$. In one patient NT-proBNP had not been determined and one patient died prior to a complete assessment. Seven patients refused to participate in the study. In the remaining 121 patients, noninvasive diagnostic procedures were performed in an outpatient setting. Based on the diagnostic algorithm, each patient was categorised as "precapillary PH excluded" or "pre-capillary PH likely". In a next step, all patients underwent invasive haemodynamic assessment by RHC. Based on these results, a diagnosis was made that served as the validation standard.

\section{Transthoracic echocardiography}

All TTE studies were performed by board certified physicians in the echo laboratory of the Medical University of Vienna using high-end scanners, such as Acuson Sequoia (Siemens AG, Erlangen, Germany) and Vivid 5 and Vivid 7 (General Electric Medical System, Milwaukee, WI, USA). The current standard in our echo lab is that all readings are performed by two independent observers. TTE studies were based on measurements that are broadly available and routinely used in the evaluation of patients with suspected $\mathrm{PH}$.

Right ventricular dysfunction was diagnosed based on a visual assessment and on a tricuspid annular plane systolic excursion of $<18 \mathrm{~mm}$. Ppa,sys was calculated by adding estimated right atrial pressure to the tricuspid regurgitation pressure gradient. No contrast agents were used for enhancement of Doppler signals. Right atrial pressure was estimated based on the diameter and respiratory variation of the inferior vena cava [11].

Left ventricular hypertrophy was diagnosed if end-diastolic septal thickness in the apical four-chamber view was $\geqslant 12 \mathrm{~mm}$.

Left ventricular diastolic dysfunction was diagnosed in the presence of a restrictive or pseudo-normal filling pattern and normal or only mildly abnormal left ventricular ejection fraction $(>50 \%)$. A restrictive filling pattern was defined by an E/A waves ratio $>2$ and a deceleration time $<150 \mathrm{~ms}$. A pseudo-normal filling pattern was diagnosed if E/A waves ratio and deceleration time were normal but changed to abnormal after Valsalva [24].

\section{ECG}

Retrospective and prospective study

A 12-lead ECG was recorded according to clinical standards at a paper speed of $25 \mathrm{~mm} \cdot \mathrm{s}^{-1}$. ECGs were analysed by two cardiologists who were blinded to the clinical and echocardiographic data. ECG rulers and callipers were used. In case of disagreement, consensus was achieved between the two observers in a second reading. The following parameters were obtained: 1) presence or absence of sinus rhythm; 2) heart rate in beats per min; 3) P-wave amplitude in $\mathrm{mV}$ in lead II; 4) $\mathrm{P}$-wave axis in degrees; 5 ) presence or absence of P-pulmonale defined as a P-wave amplitude $>0.25 \mathrm{mV}$; 6) electric heart axis in degrees; 7) right axis deviation defined as a QRS axis $>110^{\circ}$; 8) QRS width in ms; 9) presence or absence of bundle branch block defined as a QRS width $>100 \mathrm{~ms}$, and stratification in right bundle branch block or left bundle branch block; 10) the presence or absence of RVS pattern defined as ST-segment deviation and T-wave inversion in leads V1-V3 [25]; 11) the presence or absence of left ventricular strain pattern defined as ST-segment deviation and T-wave inversions in leads V5 and V6; 12) QT-length in ms and corrected QT-length calculated by the Bazett formula [26]. RVH was defined by a ratio of R and S in lead $\mathrm{V} 1>1$. 


\section{Assessments}

A detailed medical history including medical conditions known to be associated with $\mathrm{PH}$ was obtained [2, 5, 6, 27]. Associated conditions were collagen vascular disease, HIV infection, history of appetite suppressant intake, splenectomy or ventriculo-atrial shunt. In addition, exercise capacity measured by the distance in meters walked in $6 \mathrm{~min}$, and the modified New York Heart Association (NYHA) class [28] at presentation were determined in each patient. Blood gas analysis was performed using a ABL 510 blood gas analyser (Radiometer Medical ApS, Brønshøj, Denmark).

Serum NT-proBNP was measured utilising the Elecsys proBNP kit (Roche, Basel, Switzerland). Haemodynamic assessment by RHC included measurement of cardiac output utilising both the Fick equation and the thermodilution method. Pre-capillary $\mathrm{PH}$ was diagnosed if mean pulmonary arterial pressure $\left(\bar{P}_{\mathrm{pa}}\right)$ exceeded $25 \mathrm{mmHg}$ at rest, and pulmonary capillary wedge pressure was $<15 \mathrm{mmHg}$ [2].

\section{Statistical analysis}

Statistical computations were performed with SPSS (version 15.0; SPSS Inc., Chicago, IL, USA) and SAS (version 9.1; SAS Institute Inc., Cary, NC, USA). Continuous variables were described with mean and SD. Groups were compared using the unpaired t-test. Right-skewed variables were logarithmically transformed before testing. Nominal variables were described with counts and percentages, groups were compared with Chisquared or Fisher's exact test. Wilson's method was used to compute confidence intervals (CI) for single proportions. McNemar's test was used to compare sensitivities and specificities of diagnostic decision rules. All reported p-values are the result of two-tailed tests, and p-values $<0.05$ were considered statistically significant.

Univariable logistic regression models were used to assess whether TTE, clinical and ECG variables allowed discrimination between pre-capillary $\mathrm{PH}$ and no pre-capillary PH. Stepwise selection (forward search) within the three groups (TTE, clinical and ECG variables) yielded partially independent variable sets. The clinical and the ECG variable sets were used to construct a diagnostic decision tree (Classification and Regression Tree; CART) for pre-capillary PH versus no precapillary PH employing the CHAID (Chi-Squared Automatic Interaction Detection) method of SPSS and defining diagnostic branch points and terminal nodes. At each branch point a case will either branch to the left or to the right based on a test against a threshold predictor value, and will continue branching in subsequent nodes until a terminal node is reached.

Because of the prognostic implications of a delayed PAH diagnosis and treatment [1], a false negative diagnosis was assumed to have far more serious consequences than a false positive one. Therefore, the class assignment rule was chosen in a way that the percentage of false negative predictions did not exceed $4 \%$ (one out of 25 ) of the true positive cases.

To overcome the problem of overoptimistic results, both an internal and a temporal validation step were added [29]. Internal validation was based on the bootstrap approach [30], reporting an average of 30 bootstrap samples. Temporal validation was based on the prospective sample.

\section{RESULTS}

\section{Characteristics of the retrospective patient cohort}

Patient characteristics are summarised in table 1. According to RHC results, 187 (74.5\%) patients were classified as pre-capillary $\mathrm{PH}$ and $64(25.5 \%)$ as no pre-capillary $\mathrm{PH}$.

In the pre-capillary $\mathrm{PH}$ group, 49 patients were eventually diagnosed with idiopathic or familial $\mathrm{PH}$, or $\mathrm{PH}$ associated with corrected or small $(<2 \mathrm{~cm})$ uncorrected atrial septal defects, two patients had a history of anorexigen intake, six were HIVpositive, 10 female patients suffered from collagen vascular disease, two had $\mathrm{PH}$ in association with Osler's disease, 13 patients had underlying lung disease [31], 97 patients had CTEPH, 7 had porto-pulmonary hypertension, and one patient suffered from pulmonary veno-occlusive disease.

In the no pre-capillary $\mathrm{PH}$ group, 53 patients were diagnosed with post-capillary $\mathrm{PH}$. Of those, 42 patients suffered from $\mathrm{PH}$ due to left ventricular diastolic dysfunction (34 with systemic arterial hypertension and 10 with significant coronary artery disease). Nine patients with post-capillary hypertension suffered from $\mathrm{PH}$ due to lung disease and/or hypoxaemia [32]. In 11 patients $\mathrm{PH}$ was excluded because $\bar{P}_{\text {pa }}$ was $<25 \mathrm{mmHg}$. Of those, one patient was diagnosed with pulmonary lymphangioleiomyomatosis, one patient had an isolated pulmonary $\mathrm{AV}$ malformation, two patients had manifest hyperthyreoidism, two patients were diagnosed with unilateral pulmonary artery occlusion, two patients had atrial septal defect and three patients had severe isolated tricuspid valve regurgitation.

There were no between-group differences with respect to demographic characteristics, including age and sex. Furthermore, no differences were encountered in the 6-min walking distances or serum creatinine levels. However, statistically significant differences between pre-capillary $\mathrm{PH}$ and no pre-capillary $\mathrm{PH}$ were found in NYHA class, serum NT-proBNP, $\mathrm{Pa}_{2} \mathrm{CO}_{2}$, associated medical conditions, TTE parameters (e.g. Ppa,sys, right ventricular function and diameter, left ventricular wall thickness and diastolic function), haemodynamic parameters, heart rate, and ECG characteristics, e.g. P-wave amplitude and axis, QRS axis, RVH, bundle branch block, RVS pattern and the corrected QT interval (table 1).

\section{Characteristics of the prospective patient cohort}

Of 64 (52.9\%) patients diagnosed with pre-capillary PH (table 2), 26 had CTEPH, 18 idiopathic or familial $\mathrm{PH}$ or $\mathrm{PH}$ associated with corrected or small uncorrected atrial septal defect, 13 suffered from $\mathrm{PH}$ associated with chronic lung disease [31], three had porto-pulmonary hypertension, two suffered from $\mathrm{PH}$ in association with collagen vascular disease and two had $\mathrm{PH}$ associated with HIV infection.

Of the 57 (47.1\%) patients diagnosed with no pre-capillary $\mathrm{PH}$, 32 suffered from post-capillary $\mathrm{PH}$ associated with either chronic lung disease [32] or diastolic left ventricular dysfunction, and 25 had normal pulmonary pressures [16]. Patients with normal pulmonary pressures suffered from parenchymal or bronchial pulmonary diseases $(n=9)$, collagen vascular disease $(n=3)$, coronary artery disease $(n=2)$, unilateral occlusion of the pulmonary artery $(n=1)$, isolated tricuspid regurgitation $(n=1)$, patent arterial duct $(n=1)$ or abnormal pulmonary vein drainage $(n=1)$, and seven patients suffered from isolated systemic hypertension. 
TABLE 1 Retrospective clinical, echocardiographic, haemodynamic and electrocardiographic characteristics of patients with pre-capillary pulmonary hypertension $(\mathrm{PH})$ and no pre-capillary $\mathrm{PH}$

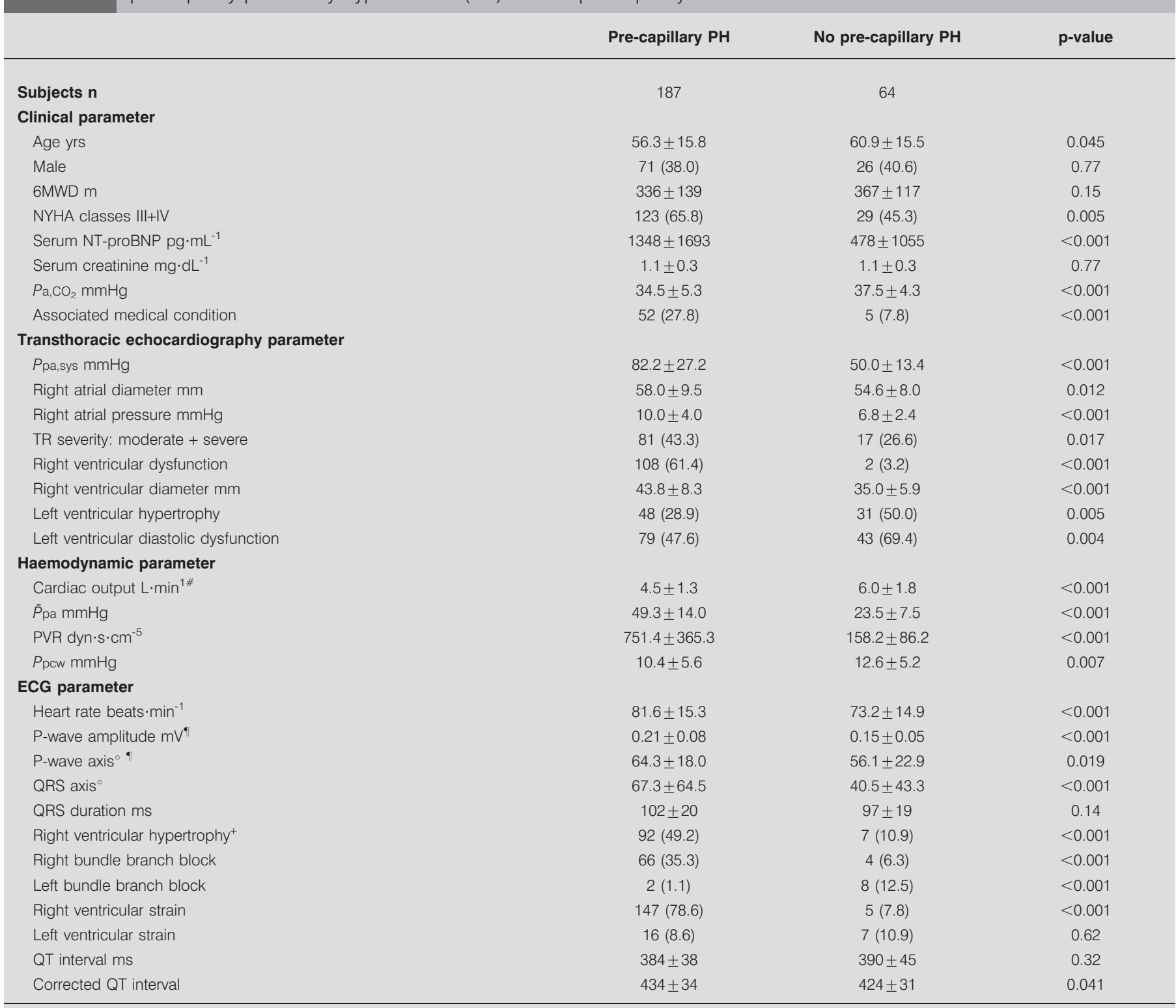

Data are presented as mean $\pm \mathrm{SD}$ or $\mathrm{n}(\%)$, unless otherwise stated. 6MWD: 6-min walk distance; NYHA: New York Heart Association; NT-proBNP: N-terminal brain natriuretic peptide; $\mathrm{Pa}_{1} \mathrm{CO}_{2}$ : arterial carbon dioxide tension; $P$ pa,sys: systolic pulmonary artery pressure; TR: tricuspid valve regurgitation; $\bar{P}$ pa: mean pulmonary artery pressure; PVR: pulmonary vascular resistance; Ppcw; pulmonary capillary wedge pressure. ${ }^{\#}$ : derived from measurements based on the thermodilution method; ": 19 patients were excluded from $\mathrm{p}$-wave analysis because of atrial fibrillation; ${ }^{+}$: diagnosed when the ratio of $\mathrm{R}$ and $\mathrm{S}$ in lead V1 was $>1$.

\section{Predictors of diagnosis}

Based on retrospective patient data, univariable (table 3) and three separate multivariable logistic regression models (table 4) were constructed for TTE, clinical parameters and ECG variables. Ppa,sys (OR 1.06, 95\% CI 1.03-1.09; p<0.001), right ventricular dysfunction (OR 10.28, 95\% CI 2.18-48.44; $p=0.003$ ) and the absence of left ventricular hypertrophy (OR $0.34,95 \%$ CI $0.15-0.75 ; \mathrm{p}=0.008$ ) were identified as independent predictors of pre-capillary $\mathrm{PH}$. Of the clinical variables tested, serum NT-proBNP (OR 2.01, 95\% CI; $\mathrm{p}=0.007), \mathrm{Pa}_{\mathrm{a}} \mathrm{CO}_{2}(\mathrm{OR} 0.86,95 \% \mathrm{CI} 0.79-0.93 ; \mathrm{p}<0.001)$ and associated medical conditions (OR 3.37, 95\%CI 1.04-10.90; $\mathrm{p}=0.043$ ) remained independent discriminative factors. ECG variables with the strongest diagnostic accuracy were heart rate (OR 1.05, 95\% CI 1.02-1.08; $\mathrm{p}<0.001$ ) and RVS (OR 52.93, 95\% CI 17.27-162.18; $\mathrm{p}<0.001)$.

\section{Accuracy of CART}

Because all patients with $P$ pa,sys $\geqslant 36 \mathrm{mmHg}$ and a suspicion of $\mathrm{PH}$ were referred for RHC, the sensitivity of TTE was $100 \%$ 


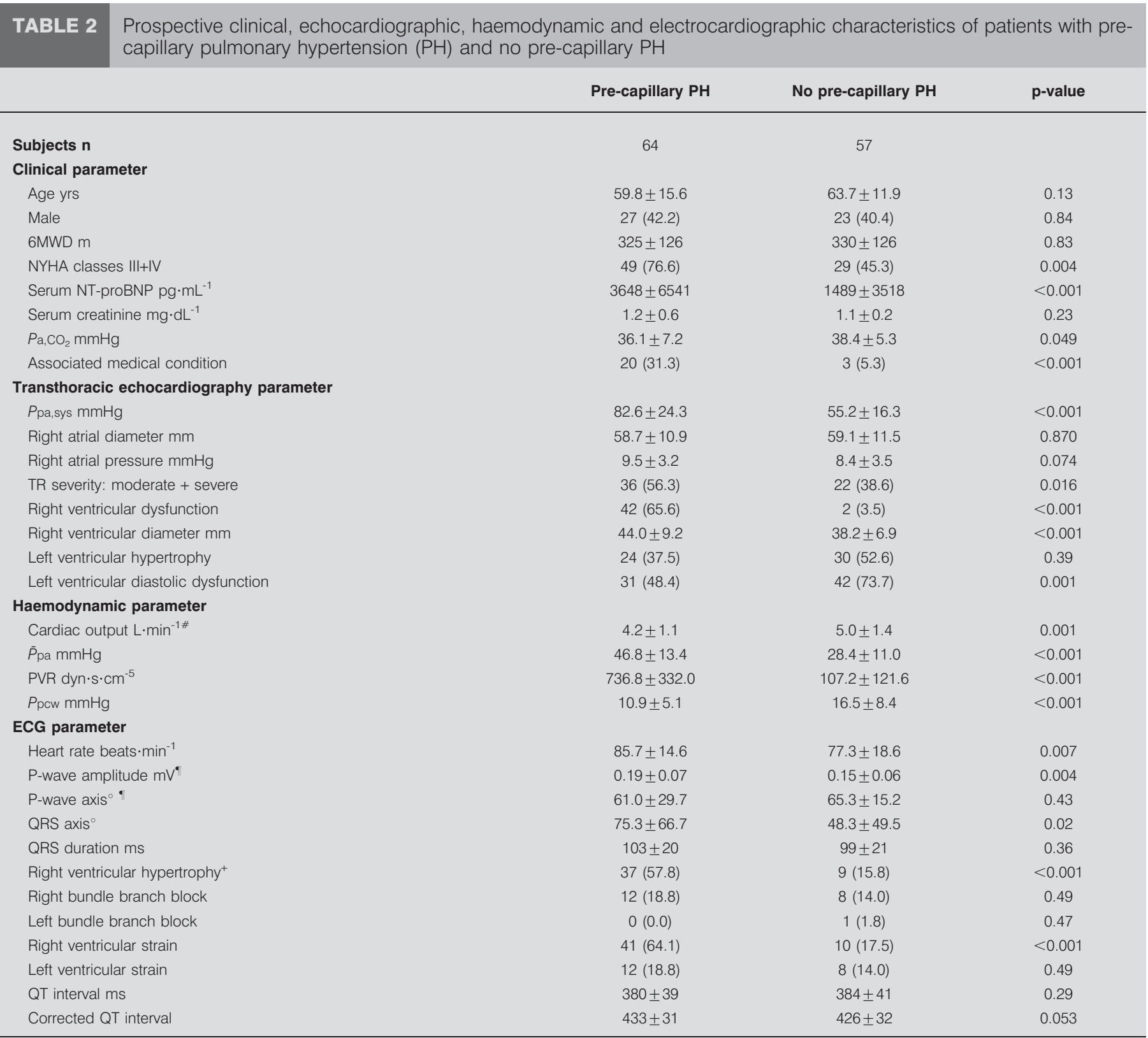

Data are presented as mean \pm standard deviation or $n(\%)$, unless otherwise stated. 6MWD: 6-min walk distance; NYHA: New York Heart Association; NT-proBNP: $\mathrm{N}$-terminal brain natriuretic peptide; $\mathrm{Pa}_{1} \mathrm{CO}_{2}$ : arterial carbon dioxide tension; $\mathrm{Ppa}$,sys: systolic pulmonary artery pressure; TR: tricuspid valve regurgitation; $\bar{P}$ pa: mean pulmonary artery pressure; PVR: pulmonary vascular resistance; $P$ pcw; pulmonary capillary wedge pressure. ${ }^{*}$ : derived from measurements based on the thermodilution method; " : 27 patients were excluded from p-wave analysis because of atrial fibrillation; ${ }^{+}$: diagnosed when the ratio of $\mathrm{R}$ and $\mathrm{S}$ in lead V1 was $>1$.

and the specificity was $0.0 \%$. For a more specific noninvasive diagnostic decision tree, all independent clinical and ECG parameters that had been identified in the multivariable logistic regression models (table 4) were fed into the CART algorithm. The model automatically selected RVS and NT-proBNP for primary decision tree construction (fig. 1).

CART had a sensitivity of $99.4 \%$ and a specificity of $40.7 \%$. Because these figures may be too optimistic as they are computed from data the tree had been derived from, two validation steps were added. Internal validation by the bootstrap technique was performed on the original data set resulting in a sensitivity of $97.9 \%$ (95\% CI 94.5-99.2\%) and a specificity of $17.3 \%$ (95\% CI 9.5-29.5\%). Temporal validation was performed on data from 121 prospectively recruited patients and yielded a sensitivity of 100\% (95\% CI 94.3-100\%) and a specificity of $19.3 \%$ (95\%CI: $11.1-31.3 \%$ ) (table 5). While sensitivity was unchanged $(100 \%$ versus $100 \%$; $p=1.0)$, specificity had improved from $0.0 \%$ according to current clinical practice to $19.3 \%(p=0.0009)$. 


\begin{tabular}{|c|c|c|c|}
\hline \multirow[t]{2}{*}{ TABLE 3} & \multicolumn{3}{|c|}{$\begin{array}{l}\text { Clinical, echocardiographic and ECG } \\
\text { parameters to predict pre-capillary pulmonary } \\
\text { hypertension in the retrospective cohort } \\
\text { (univariable logistic regression) }\end{array}$} \\
\hline & & OR $(95 \% \mathrm{Cl})$ & p-value \\
\hline \multicolumn{4}{|c|}{ Clinical parameter } \\
\hline Age yrs & & $0.98(0.96-1.00)$ & 0.047 \\
\hline Male & & $0.89(0.50-1.600$ & 0.71 \\
\hline $6 \mathrm{MWD} \mathrm{km}$ & & $0.17(0.02-1.93)$ & 0.15 \\
\hline NYHA clas & III+IV & $2.32(1.30-4.13)$ & 0.004 \\
\hline Serum NT- & $o B N P \mathrm{ng} \cdot \mathrm{mL}^{-1 \#}$ & $2.05(1.31-3.22)$ & 0.002 \\
\hline $\mathrm{Pa}, \mathrm{CO}_{2} \mathrm{~mm}$ & & $0.89(0.84-0.95)$ & $<0.001$ \\
\hline Associated & nedical condition & $4.55(1.73-11.96)$ & 0.002 \\
\hline \multicolumn{4}{|c|}{ TTE parameter } \\
\hline Ppa,sys mm & & $1.08(1.05-1.10)$ & $<0.001$ \\
\hline Right atrial & iameter $\mathrm{mm}$ & $1.04(1.01-1.08)$ & 0.0113 \\
\hline Right atrial & ressure $\mathrm{mmHg}$ & $1.32(1.19-1.47)$ & $<0.001$ \\
\hline TR severity & noderate + severe & $2.36(1.26-4.42)$ & 0.008 \\
\hline Right ventri & ular dysfunction & $48.44(11.47-204.61)$ & $<0.001$ \\
\hline Right ventr & ular diameter $\mathrm{mm}$ & $1.18(1.12-1.24)$ & $<0.001$ \\
\hline Left ventric & ar hypertrophy & $0.41(0.22-0.74)$ & 0.003 \\
\hline Left ventric & ar diastolic dysfunction & $0.40(0.22-0.75)$ & 0.004 \\
\hline \multicolumn{4}{|c|}{ ECG parameter } \\
\hline Heart rate & eats $\cdot \min ^{-1}$ & $1.04(1.02-1.06)$ & $<0.001$ \\
\hline P-wave am & litude $\mu \mathrm{V}^{\#}$ & $1.01(1.01-1.02)$ & $<0.001$ \\
\hline P-wave axi & & $1.02(1.01-1.04)$ & 0.009 \\
\hline QRS axis $^{\circ}$ & & $1.01(1.00-1.01)$ & 0.003 \\
\hline QRS durati & ns $\mathrm{ms}$ & $1.01(1.00-1.03)$ & 0.14 \\
\hline Right ventri & ular hypertrophy & 7.89 (3.42-18.19) & $<0.001$ \\
\hline Right bund & branch block & $8.18(2.85-23.51)$ & $<0.001$ \\
\hline Left bundle & ranch block & $0.08(0.02-0.37)$ & 0.001 \\
\hline Right ventr & ular strain & $43.37(16.32-115.26)$ & $<0.001$ \\
\hline Left ventric & ar strain & $0.76(0.30-1.95)$ & 0.57 \\
\hline Corrected & T interval & $1.01(1.00-1.02)$ & 0.043 \\
\hline
\end{tabular}

6MWD: 6-min walk distance; NYHA: New York Heart Association; NT-proBNP: $\mathrm{N}$-terminal brain natriuretic peptide; $\mathrm{Pa}_{\mathrm{a}} \mathrm{CO}_{2}$ : arterial carbon dioxide tension; TTE: transthoracic echocardiography; $P$ pa,sys: systolic pulmonary artery pressure; TR: tricuspid valve regurgitation. ${ }^{*}$ : units of measurement were adjusted for better readability of confidence intervals.

\section{DISCUSSION}

According to current guidelines [2], a significant proportion of patients referred for unexplained dyspnoea are undergoing invasive pressure measurements. To test the accuracy of standard noninvasive diagnostic tools, we analysed data sets from 251 retrospective and 121 prospective patients who underwent RHC for evaluation of PH. The main finding of our study was that ECG and NT-proBNP in addition to TTE suffice to predict significant pre-capillary pulmonary vascular disease at a level of sensitivity of $100 \%$ and specificity of $19.3 \%$. In practice, based on the CART algorithm, RHC can be safely withheld in $9 \%$ of patients with elevated echo Ppa,sys and clinical PH suspicion without overlooking true $\mathrm{PH}$ cases.

$\mathrm{PAH}$, which is one of the major causes of pre-capillary $\mathrm{PH}$, is a severe condition with serious prognosis [33]. The availability of potent vasodilator therapies that positively impact on morbidity

\begin{tabular}{|c|c|c|c|}
\hline \multirow[t]{2}{*}{ TABLE 4} & \multicolumn{3}{|c|}{$\begin{array}{l}\text { Clinical, echocardiographic and ECG } \\
\text { parameters to predict pre-capillary pulmonary } \\
\text { hypertension in the retrospective cohort } \\
\text { (multivariable logistic regression) }\end{array}$} \\
\hline & & OR (95\% Cl) & p-value \\
\hline \multicolumn{4}{|c|}{ Clinical parameter } \\
\hline \multicolumn{2}{|c|}{ Serum NT-proBNP ng $\cdot \mathrm{mL}^{-1 \#}$} & $2.01(1.21-3.33)$ & 0.007 \\
\hline \multicolumn{2}{|c|}{$\mathrm{Pa}, \mathrm{CO}_{2} \mathrm{mmHg}$} & $0.86(0.79-0.93)$ & $<0.001$ \\
\hline \multicolumn{2}{|c|}{ Associated medical condition } & $3.37(1.04-10.90)$ & 0.043 \\
\hline \multicolumn{4}{|c|}{ TTE parameter } \\
\hline \multicolumn{2}{|c|}{ Ppa,sys $\mathrm{mmHg}$} & $1.06(1.03-1.09)$ & $<0.001$ \\
\hline \multicolumn{2}{|c|}{ Right ventricular dysfunction } & $10.28(2.18-48.44)$ & 0.003 \\
\hline \multicolumn{2}{|c|}{ Left ventricular hypertrophy } & $0.34(0.15-0.75)$ & 0.008 \\
\hline \multicolumn{4}{|c|}{ ECG parameter } \\
\hline \multicolumn{2}{|c|}{ Heart rate beats $\cdot \mathrm{min}^{-1}$} & $1.05(1.02-1.08)$ & $<0.001$ \\
\hline \multicolumn{2}{|c|}{ Right ventricular strain } & $52.93(17.27-162.18)$ & $<0.001$ \\
\hline
\end{tabular}

NT-proBNP: N-terminal brain natriuretic peptide; $\mathrm{Pa}_{\mathrm{a}} \mathrm{CO}_{2}$ : arterial carbon dioxide tension; TTE: transthoracic echocardiography; Ppa,sys: systolic pulmonary artery pressure. ${ }^{\#}$ : units of measurement were adjusted for better readability of confidence intervals.

and mortality $[2,34]$ has supported early recognition and treatment [20, 35]. Moreover, screening of asymptomatic individuals at increased risk for $\mathrm{PH}$ has been recommended. While invasive haemodynamic assessment has been considered the diagnostic gold standard in PH, TTE is the recommended screening tool [20,34]. TTE is noninvasive, broadly available and most studies report a high correlation of $0.57-0.93$ between TTE and invasive measurements of pulmonary arterial systolic pressures [2]. However, elevated $P$ pa,sys may result from either pre-capillary $\mathrm{PH}$ or post-capillary $\mathrm{PH}$, which cannot be safely distinguished by TTE alone. In contrast to post-capillary PH

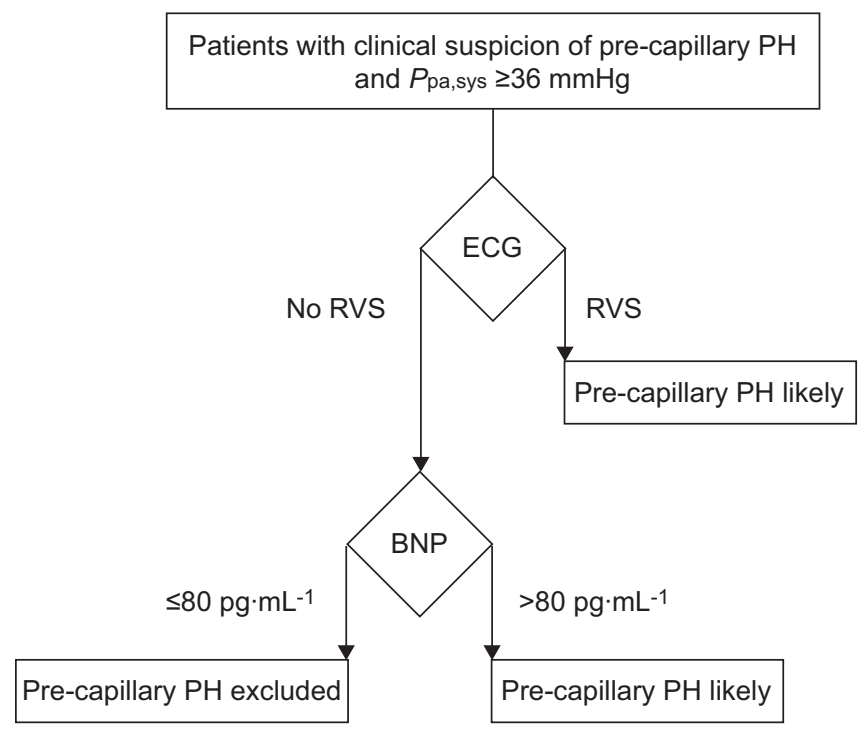

FIGURE 1. Pulmonary hypertension $(\mathrm{PH})$ diagnosis tree based on the Classification and Regression Tree algorithm. Ppa,sys: systolic pulmonary artery pressure; BNP: brain natriuretic peptide; RVS: right ventricular strain. 
TABLE 5 Prospective validation of the Classification and Regression Tree algorithm

\begin{tabular}{lcccc} 
Pre-capillary PH diagnosed by RHC & RVS & $\begin{array}{c}\text { No RVS and NT-proBNP } \\
>\mathbf{8 0} \mathbf{~ p g} \cdot \mathbf{m L}^{-1}\end{array}$ & $\begin{array}{c}\text { No RVS and NT-proBNP } \\
\leqslant \mathbf{8 0 ~} \mathbf{~ g ~} \cdot \mathbf{m L}^{-1}\end{array}$ \\
\hline Yes & 41 & 23 & 0 & Total \\
No & 11 & 35 & 11 & 64 \\
Total & 52 & 58 & 11 & 121 \\
\hline
\end{tabular}

Data are presented as $\mathrm{n}$ and results reflect the true predictive power of the decision tree because the prospectively recruited patients did not contribute to decision tree construction. A total of 121 patients were studied. Right ventricular strain (RVS) pattern was present in 52 patients, 41 of whom were diagnosed with pre-capillary pulmonary hypertension $(\mathrm{PH})$ by right heart catheter $(\mathrm{RHC})$. Patients without RVS pattern on $E C G(n=69)$ were further dichotomised according to serum $\mathrm{N}$-terminal brain natriuretic peptide (NT-proBNP) levels. 58 patients displayed serum NT-proBNP levels $>80 \mathrm{pg} \cdot \mathrm{mL}^{-1}$; of these 23 were diagnosed with pre-capillary PH. In contrast, none of the 11 patients with serum NT-proBNP $\leqslant 80 \mathrm{pg} \cdot \mathrm{mL}^{-1}$ were diagnosed with pre-capillary PH.

[15], pre-capillary vascular disease is a rare condition [7]. These circumstances have recently led to numerous invasive procedures for exclusion. To narrow the grey zone that is blurring the distinction between pre- and post-capillary disease by echocardiography, current recommendations propose an invasive diagnostic work-up in patients with echocardiographic $P$ pa,sys values $>50 \mathrm{mmHg}$ [36]. According to our assessments, this diagnostic approach would have substantially increased specificity to $42.1 \%$; however, at the cost of overlooking $6.2 \%$ of true pre-capillary $\mathrm{PH}$ cases.

Although of limited value, standard ECG has diagnostic [37] and prognostic [38] potential in PH. Despite the fact that postcapillary PH may cause an RVS pattern on ECG [39], RVS remained the strongest predictor of pre-capillary $\mathrm{PH}$ in our study. RVS depicts right ventricular electric repolarisation, and appears to be a sensitive and immediate marker of right ventricular strain. The presence of RVS correctly identified $78.8 \%$ of pre-capillary $\mathrm{PH}$ cases. In addition, several noninvasive prognostic parameters have been established over the years, e.g. the 6-min walk distance [40], NYHA functional class [1], $\mathrm{Pa}, \mathrm{CO}_{2}$ [23], associated medical conditions [27, 41-43], and serum NT-proBNP levels [44]. NT-proBNP is released from both cardiac ventricles in response to increased wall tension and is elevated in $\mathrm{PH}$ correlating well with pulmonary vascular resistance [45]. Its discriminative accuracy with respect to cardiac versus noncardiac causes of dyspnoea has been described previously [46], and is primarily based upon its excellent negative predictive value of $96 \%$ [47]. The combination of RVS and NT-proBNP, one being a strong positive, the other a strong negative predictor, confers clinical usefulness.

A main limitation of our study is its single centre design. A centre-specific bias with respect to diagnostic procedures, in particular TTE, cannot be excluded. Different referral patterns may influence proportions of $\mathrm{PH}$ versus non-PH cases. However, in contrast to positive and negative predictive values, sensitivities and specificities for the detection of $\mathrm{PH}$ are independent of the prevalence of healthy individuals in a given cohort.

Taken together, we would like to emphasise that RHC remains the gold standard for the evaluation of PH [11-13]. However, it is widely accepted that a diagnostic procedure that is primarily undertaken to rule out a disease is ideally noninvasive. In a selected patient population referred for dyspnoea and echocardiographic suspicion of $\mathrm{PH}$, integration of the decision tree subsequent to TTE helps to avoid unnecessary RHCs in $9 \%$ of cases while not missing a single true $\mathrm{PH}$ case.

\section{SUPPORT STATEMENT}

This study received financial support from by the European Commission under the 6th Framework Programme (Contract No LSHM-CT-2005-018725, PULMOTENSION to I.M. Lang). This publication reflects only the author's views and the European Community is in no way liable for any use that may be made of the information contained therein. The research was supported by the Austrian fellowship grant FWFL 513-B11 to D. Bonderman and the Hans und Blanca Moser Stiftung to A.M. Martischnig.

\section{STATEMENT OF INTEREST}

None declared.

\section{REFERENCES}

1 D'Alonzo GE, Barst RJ, Ayres SM, et al. Survival in patients with primary pulmonary hypertension. Results from a national prospective registry. Ann Intern Med 1991; 115: 343-349.

2 Galie N, Torbicki A, Barst R, et al. Guidelines on diagnosis and treatment of pulmonary arterial hypertension. The Task Force on Diagnosis and Treatment of Pulmonary Arterial Hypertension of the European Society of Cardiology. Eur Heart J 2004; 25: 2243-2278.

3 Abenhaim L, Moride Y, Brenot F, et al. Appetite-suppressant drugs and the risk of primary pulmonary hypertension. International Primary Pulmonary Hypertension Study Group. N Engl J Med 1996; 335: 609-616.

4 Petitpretz P, Brenot F, Azarian R, et al. Pulmonary hypertension in patients with human immunodeficiency virus infection. Comparison with primary pulmonary hypertension. Circulation 1994; 89: 2722-2727.

5 Bonderman D, Jakowitsch J, Adlbrecht C, et al. Medical conditions increasing the risk of chronic thromboembolic pulmonary hypertension. Thromb Haemost 2005; 93: 512-516.

6 Bonderman D, Wilkens H, Wakounig S, et al. Risk factors for chronic thromboembolic pulmonary hypertension. Eur Respir J 2009; 33: 325-331.

7 Gaine SP, Rubin LJ. Primary pulmonary hypertension. Lancet 1998; 352: 719-725.

8 Bonderman D, Jakowitsch J, Redwan B, et al. Role for staphylococci in misguided thrombus resolution of chronic thromboembolic pulmonary hypertension. Arterioscler Thromb Vasc Biol 2008; 28: 678-684. 
9 Jais X, Ioos V, Jardim C, et al. Splenectomy and chronic thromboembolic pulmonary hypertension. Thorax 2005; 60: 1031-1034.

10 Lang IM. Chronic thromboembolic pulmonary hypertension: not so rare after all. N Engl J Med 2004; 350: 2236-2238.

11 Badesch DB, Champion HC, Sanchez MA, et al. Diagnosis and assessment of pulmonary arterial hypertension. J Am Coll Cardiol 2009; 54: Suppl. 1, S55-S66.

12 Galie N, Hoeper MM, Humbert M, et al. Guidelines for the diagnosis and treatment of pulmonary hypertension. Eur Respir $J$ 2009; 34: 1219-1263.

13 Galie N, Hoeper MM, Humbert M, et al. Guidelines for the diagnosis and treatment of pulmonary hypertension: The Task Force for the Diagnosis and Treatment of Pulmonary Hypertension of the European Society of Cardiology (ESC) and the European Respiratory Society (ERS), endorsed by the International Society of Heart and Lung Transplantation (ISHLT). Eur Heart J 2009; 30: 2493-2537.

14 Hoeper MM, Barbera JA, Channick RN, et al. Diagnosis, assessment, and treatment of non-pulmonary arterial hypertension pulmonary hypertension. J Am Coll Cardiol 2009; 54: Suppl. 1, S85-S96.

15 Paulus WJ, Tschope C, Sanderson JE, et al. How to diagnose diastolic heart failure: a consensus statement on the diagnosis of heart failure with normal left ventricular ejection fraction by the Heart Failure and Echocardiography Associations of the European Society of Cardiology. Eur Heart J 2007; 28: 2539-2550.

16 Fisher MR, Forfia PR, Chamera E, et al. Accuracy of Doppler echocardiography in the hemodynamic assessment of pulmonary hypertension. Am J Respir Crit Care Med 2009; 179: 615-621.

17 Michelakis ED. Soluble guanylate cyclase stimulators as a potential therapy for PAH: enthusiasm, pragmatism and concern. Eur Respir J 2009; 33: 717-721.

18 Hoeper MM, Lee SH, Voswinckel R, et al. Complications of right heart catheterization procedures in patients with pulmonary hypertension in experienced centers. J Am Coll Cardiol 2006; 48: 2546-2552.

19 Schenk P, Stiebellehner L, Burghuber OC, et al. [Examination of pulmonary circulation using right heart catheterization. Position paper of the Cardiopulmonary Task Force of the Austrian Society for Pneumology and the Austrian Society for Cardiology]. Wien Klin Wochenschr 2005; 117: 651-662.

20 McGoon M, Gutterman D, Steen V, et al. Screening, early detection, and diagnosis of pulmonary arterial hypertension: ACCP evidencebased clinical practice guidelines. Chest 2004; 126: Suppl. 1, 14S-34S.

21 Rich S, Dantzker DR, Ayres SM, et al. Primary pulmonary hypertension. A national prospective study. Ann Intern Med 1987; 107: 216-223.

22 Fijalkowska A, Kurzyna M, Torbicki A, et al. Serum N-terminal brain natriuretic peptide as a prognostic parameter in patients with pulmonary hypertension. Chest 2006; 129: 1313-1321.

23 Hoeper MM, Pletz MW, Golpon H, et al. Prognostic value of blood gas analyses in patients with idiopathic pulmonary arterial hypertension. Eur Respir J 2007; 29: 944-950.

24 Dickstein K, Cohen-Solal A, Filippatos G, et al. ESC Guidelines for the diagnosis and treatment of acute and chronic heart failure 2008: the Task Force for the Diagnosis and Treatment of Acute and Chronic Heart Failure 2008 of the European Society of Cardiology. Developed in collaboration with the Heart Failure Association of the ESC (HFA) and endorsed by the European Society of Intensive Care Medicine (ESICM). Eur Heart J 2008; 29: 2388-2442.

25 Braunwald E. Braunwald's Heart Disease: A Textbook of Cardiovascular Medicine. 5th Edn. Philadelphia, Elsevier Health, 2004.

26 Burchell HB. The QT interval historically treated. Pediatr Cardiol 1983; 4: 139-148.

27 Bonderman D, Skoro-Sajer N, Jakowitsch J, et al. Predictors of outcome in chronic thromboembolic pulmonary hypertension. Circulation 2007; 115: 2153-2158.
28 Hoeper MM, Oudiz RJ, Peacock A, et al. End points and clinical trial designs in pulmonary arterial hypertension: clinical and regulatory perspectives. J Am Coll Cardiol 2004; 43: Suppl. 12, 48S-55S.

29 Koenig IR, Malley JD, Weimar C, et al. Practical experiences on the necessity of external validation. Statist Med 2007; 26: 5499-5511.

30 Efron B, Tibshirani RJ. An Introduction to the Bootstrap. New York, Chapman and Hall, 1993.

31 Weitzenblum E, Chaouat A, Canuet M, et al. Pulmonary hypertension in chronic obstructive pulmonary disease and interstitial lung diseases. Semin Respir Crit Care Med 2009; 30: 458-470.

32 Funk GC, Lang I, Schenk P, et al. Left ventricular diastolic dysfunction in patients with COPD in the presence and absence of elevated pulmonary arterial pressure. Chest 2008; 133: 1354-1359.

33 Humbert M, Sitbon O, Chaouat A, et al. Pulmonary arterial hypertension in France: results from a national registry. Am J Respir Crit Care Med 2006; 173: 1023-1030.

34 Grunig E, Weissmann S, Ehlken N, et al. Stress Doppler echocardiography in relatives of patients with idiopathic and familial pulmonary arterial hypertension. results of a multicenter European analysis of pulmonary artery pressure response to exercise and hypoxia. Circulation 2009; 119: 1747-1757.

35 Galie N, Rubin L, Hoeper M, et al. Treatment of patients with mildly symptomatic pulmonary arterial hypertension with bosentan (EARLY study): a double-blind, randomised controlled trial. Lancet 2008; 371: 2093-2100.

36 Olschewski H, Hoeper MM, Borst MM, et al. [Diagnosis and therapy of chronic pulmonary hypertension]. Pneumologie 2006; 60: 749-771.

37 Ahearn GS, Tapson VF, Rebeiz A, et al. Electrocardiography to define clinical status in primary pulmonary hypertension and pulmonary arterial hypertension secondary to collagen vascular disease. Chest 2002; 122: 524-527.

38 Bossone E, Paciocco G, Iarussi D, et al. The prognostic role of the ECG in primary pulmonary hypertension. Chest 2002; 121: 513-518.

39 Enomoto H. Clinical studies on the ventricular strain pattern electrocardioogram (I) : clinical statistical studies. Jap Circ J 1961; 25: 1277-1286.

40 Miyamoto S, Nagaya N, Satoh $\mathrm{T}$, et al. Clinical correlates and prognostic significance of six-minute walk test in patients with primary pulmonary hypertension. Comparison with cardiopulmonary exercise testing. Am J Respir Crit Care Med 2000; 161: 487-492.

41 Kawut SM, Taichman DB, Archer-Chicko CL, et al. Hemodynamics and survival in patients with pulmonary arterial hypertension related to systemic sclerosis. Chest 2003; 123: 344-350.

42 Kawut SM, Taichman DB, Ahya VN, et al. Hemodynamics and survival of patients with portopulmonary hypertension. Liver Transpl 2005; 11: 1107-1111.

43 Rich S, Shillington A, McLaughlin V. Comparison of survival in patients with pulmonary hypertension associated with fenfluramine to patients with primary pulmonary hypertension. Am J Cardiol 2003; 92: 1366-1368.

44 Nagaya N, Nishikimi T, Uematsu M, et al. Plasma brain natriuretic peptide as a prognostic indicator in patients with primary pulmonary hypertension. Circulation 2000; 102: 865-870.

45 Pruszczyk P. N-terminal pro-brain natriuretic peptide as an indicator of right ventricular dysfunction. J Card Fail 2005; 11: Suppl. 5, S65-S69.

46 Tabbibizar R, Maisel A. The impact of B-type natriuretic peptide levels on the diagnoses and management of congestive heart failure. Curr Opin Cardiol 2002; 17: 340-345.

47 Maisel AS, Krishnaswamy P, Nowak RM, et al. Rapid measurement of B-type natriuretic peptide in the emergency diagnosis of heart failure. N Engl J Med 2002; 347: 161-167. 